Microwave and

Optical Technology Letters

\title{
Resonant optical receiver design by series inductive peaking for sub-6 GHz RoF
}

\begin{tabular}{|c|c|}
\hline Journal: & Microwave and Optical Technology Letters \\
\hline Manuscript ID & MOP-17-0161 \\
\hline Wiley - Manuscript type: & Research Article \\
\hline Date Submitted by the Author: & 03-Feb-2017 \\
\hline Complete List of Authors: & $\begin{array}{l}\text { Bogaert, Laurens; Ghent University, Department of Information Technology } \\
\text { (INTEC) } \\
\text { Van Kerrebrouck, Joris; Ghent University, Department of Information } \\
\text { Technology (INTEC) } \\
\text { Abbasi, Amin; Ghent University, Department of Information Technology } \\
\text { (INTEC) } \\
\text { Lambrecht, Joris; Ghent University, Department of Information Technology } \\
\text { (INTEC) } \\
\text { Torfs, Guy; Ghent University, Department of Information Technology } \\
\text { (INTEC) } \\
\text { Yin, Xin; Ghent University, Department of Information Technology (INTEC) } \\
\text { Roelkens, Gunther; Ghent University, Department of Information } \\
\text { Technology (INTEC) } \\
\text { Bauwelinck, Johan; Ghent University, Department of Information } \\
\text { Technology (INTEC) }\end{array}$ \\
\hline Keywords: & $\begin{array}{l}\text { Radio over Fiber, Resonant receiver, Series inductive peaking, } \\
\text { Transimpedance amplifier }\end{array}$ \\
\hline
\end{tabular}




\section{RESONANT OPTICAL RECEIVER DESIGN BY SERIES INDUCTIVE PEAKING FOR SUB-6 GHZ \\ ROF}

L. Bogaert, ${ }^{12}$ J. Van Kerrebrouck, ${ }^{1}$ A. Abbasi, ${ }^{2}$ J. Lambrecht, ${ }^{1}$ G. Torfs, ${ }^{1}$ X. Yin, ${ }^{1}$ G. Roelkens, ${ }^{2}$ and J. Bauwelinck ${ }^{1}$

${ }^{1}$ IDLab Group, Dep. INTEC, Ghent University-Imec, B-9052 Ghent, Belgium

2Photonics research group, Dep. INTEC, Ghent University-Imec, B-9052 Ghent, Belgium

Corresponding author: laurens.bogaert@ugent.be

ABSTRACT: Future wireless communication links will require ever decreasing cell sizes to provide massive data capacity densities. Implementing a fully operational base station in each cell is not viable and thus centralized solutions making use of radio-over-fiber (RoF) have been proposed.

Transimpedance amplifiers (TIA) are an essential component in such RoF links. In this article, resonant TIAs making use of series inductive peaking are proposed for RoF communication links. Such a bandpass approach benefits from superior noise behavior and a relaxation of the transimpedance limit compared to the traditional broadband designs typically used in RoF.

Key words: Radio over Fiber, resonant receiver, series inductive peaking, transimpedance amplifier 


\section{INTRODUCTION}

The ever-increasing demand for high-speed wireless data connectivity requires new wireless network infrastructure. Part of the solution for this problem is shrinking the cell sizes. To make this a viable solution, the cost per remote antenna unit (RAU) has to decrease significantly. Radio-over-fiber (RoF, [1]) makes this decrease in cost per RAU possible by using a centralized approach hereby shifting the center of gravity from the RAU to the base station. In such a way resources can be shared between multiple RAUs.

Nowadays, RoF setups typically make use of broadband electronics and are thus not yet optimized for the intended radio schemes. In this article, we focus on optimizing the receiver side of the RAU by taking into account the narrowband nature of the signal. A design approach using series inductive peaking is proposed for implementing such a resonant transimpedance amplifier (TIA). While a broadband TIA requires a lowpass behavior with sufficient bandwidth (larger than the RF carrier used in the transmission scheme), a resonant TIA only needs sufficient gain in the targeted part of the spectrum. The devised TIA is not only superior in terms of noise behavior compared to a broadband TIA but is also capable of boosting the transimpedance. Whereas the first property can also be obtained by combining the broadband TIA with a band-pass filter, the second can not.

The content of this article is subdivided in two main parts. Firstly, the resonant design approach, analytic expressions describing its behavior and its advantages over traditional designs for radio schemes are discussed. Subsequently, a proof of concept is given for sub-6 $\mathrm{GHz}$ applications. Because of the limited bandwidth available at these lower frequencies, higher order modulation formats are typically used in sub-6 GHz applications with stringent requirements on the tolerated noise levels. For these types of applications, the noise performance can be largely improved by using a resonant TIA instead of a broadband TIA because RoF typically only targets a small fraction of the spectrum that is passed by a broadband TIA.

\section{MODELING OF THE PHOTODETECTOR}


The proposed resonant TIA design process will depend heavily on the frequency dependent electrical behavior of the photodetector (PD) that is used. Since the frequency range of interest is rather small, a compact equivalent circuit (Fig. 1) can already give a good fit between simulation and measurements. The equivalent model (Fig. 1) consists of two parts. The PD itself can be modeled [2] with a current source $\left(I_{P D}\right)$, a junction capacitance $\left(C_{j}\right)$, a shunt resistance $\left(R_{s h}\right.$, which is typically negligible), and a series resistance. Secondly, the interconnect parasitics connecting the PD to the TIA are modeled as a series resistor-inductor element. This is possible because of the short interconnect length. The series resistance of the leads and the internal series resistance of the photodetector are combined in one parameter $\left(R_{s}\right)$. The inductance of the leads is denoted by $L_{\text {lead. }}$

In case of a broadband design methodology, the tolerated amount of inductance is limited (although a very small inductance value might slightly increase the bandwidth without introducing peaking [3]). For passband applications, however, the presence of the lead inductor can be utilized to our advantage to introduce high-Q peaking and favor the transfer of the desired frequency band.

\section{TRANSIMPEDANCE}

\subsection{Broadband TIA}

The transimpedance ( $\left.Z_{T}\right)$ of a broadband TIA (Fig. 2) has a low-pass behavior (1) with $3 \mathrm{~dB}$ frequency $\omega_{0, B B}(2)[4]$. These formulas were derived without taking $R_{s h}, L_{\text {lead, }}$ and $R_{s}$ into account and by using an amplifier with a frequency independent open loop voltage gain $A$. In these equations the parallel combination of the input capacitance of the TIA $\left(C_{i n}\right)$ and junction capacitance of the PD $\left(C_{j}\right)$ is combined into the total capacitance $C_{T}$ whereas $R_{F}$ denotes the feedback resistance.

$$
\begin{gathered}
Z_{T}=-\frac{A}{A+1} R_{F} \frac{1}{1+s / \omega_{0, B B}} \\
\omega_{0, B B}=\frac{A+1}{R_{F} C_{T}}(2)
\end{gathered}
$$

This low-pass behavior, although desired for baseband signals, is not needed for RoF applications. 
Resonant TIA solutions make use of this extra degree of freedom by shaping the frequency dependent gain in such a way that it improves the signal-to-noise ratio (SNR) at the desired frequencies.

\subsection{Resonant TIA}

The analytic expressions for the resonant TIA proposed in this article will be derived under the assumption of having sufficient loop gain at all frequencies of interest. Additionally, the goal is to size the amplifier and $R_{F}$ in such a way that the input resistance $R_{\text {in }}$ of the TIA (3) [4] is sufficiently smaller than the capacitive input impedance $\left|1 / \mathrm{s} C_{i n}\right|$ of the TIA. If this condition is not fulfilled around the signal band, only a (possibly small) fraction of the useful $R_{F}$ current going to the TIA will flow in the feedback path, resulting in inferior performances.

$$
R_{\text {in }} \approx \frac{R_{F}}{A+1}
$$

Under these assumptions, the transimpedance of the resonant receiver can be subdivided into two parts (4). The first part is the frequency dependent input circuitry, which will favor the desired frequency band by adopting series inductive peaking. Secondly, a current-to-voltage conversion is done through the feedback path of the TIA. This current-to-voltage conversion will by design $\left(1 / \omega C_{i n}>>R_{i n}\right.$, and frequency independent $A$ ) be assumed to be approximately frequency flat for all frequencies of interest.

$$
Z_{T}=\frac{I_{\text {in }}}{I_{P D}} \times \frac{V_{\text {out }}}{I_{\text {in }}}=\frac{I_{\text {in }}}{I_{P D}} \times-R_{F}
$$

To calculate the frequency dependent filtering from $I_{P D}$ to the current entering the $\operatorname{TIA}\left(l_{\text {in }}\right)$, the circuit depicted in Fig. 1 is loaded by the input impedance of the TIA [4](Fig. 3). When sizing the circuit correctly, it is possible to initially neglect the input capacitance for all frequencies of interest. As discussed before, this is required to make sure that most of the RF current $l_{\text {in }}$ flows through the feedback path.

Biasing the TIA and PD independently will require the use of a decouple capacitor. Its value is typically chosen in such a way that it behaves as an RF short. In Fig. 3, this DC-blocking capacitor was added explicitly to add extra tunability to the behavior of the input network. 
Since we assume the current-to-voltage conversion from the input current lin to the output voltage of the TIA to be nearly frequency flat for all frequencies of interest, the transfer function from the current generated in the PD $\left(I_{P D}\right)$ to the current entering the TIA $\left(l_{\text {in }}\right)$ will determine the frequency dependent behavior of the whole TIA.

The transfer function of the input network is easily obtained by observing that the circuit behaves as a current divider (5). The series combination of $R_{S}$ and $R_{\text {in }}$ is replaced by the total resistance $R_{T}\left(=R_{S}+R_{\text {in }}\right)$ to get a more compact expression.

$$
\frac{I_{\text {in }}}{I_{P D}}=\frac{1}{\left(1+C_{j} / C_{\text {block }}\right)+s R_{T} C_{j}+s^{2} L_{\text {lead }} C_{j}}
$$

When the decouple capacitor $C_{\text {block }}$ is realized as an ideal $D C$ block, the resonance (i.e. peaking) frequency $\omega_{0}(6)$ of this second order low-pass transfer function will depend only on $L_{\text {lead }}$ and $C_{j}$. Llead can be increased by adding an explicit inductance ( $\left.L_{\text {explicit }}\right)$ in series with the original inductance, giving rise to a decrease in resonance frequency (7).

$$
\begin{aligned}
& \omega_{0}\left(L_{\text {explicit }}=0, C_{\text {block }}=\infty\right)=\frac{1}{\sqrt{L_{\text {lead }} C_{j}}} \\
& \omega_{0}\left(C_{\text {block }}=\infty\right)=\frac{1}{\sqrt{\left(L_{\text {lead }}+L_{\text {explicit }}\right) C_{j}}}
\end{aligned}
$$

The disadvantage of using such a simple input circuit is that the product of $L_{\text {lead }}$ and $C_{j}$ will introduce an upper limit on the maximally achievable peaking frequency. This problem can be circumvented by using a finite value for $C_{\text {block }}(8)$. The parameter $\alpha\left(=\sqrt{1+C_{j} / C_{\text {block }}}\right)$ was added to describe the factor by which the resonance frequency is increased when using $C_{\text {block }}$ with a finite value.

$$
\omega_{0}=\frac{\sqrt{1+C_{j} / C_{\text {block }}}}{\sqrt{\left(L_{\text {lead }}+L_{\text {explicit }}\right) C_{j}}} \triangleq \alpha \times \omega_{0}\left(C_{\text {block }}=\infty\right)
$$


The peak height $\left|l_{\text {in }}\left(\omega_{0}\right) / l_{\text {in }}(0)\right|$ is denoted by the Quality $(Q)$-factor $(9)$. This value will have a major impact on the performance improvement of the system. With respect to the noise behavior, it is advisable to work with a high Q-factor peak as it will boost the useful band relative to the broadband noise. However, it should be stressed that a higher $Q$ also results in a lower signal bandwidth. Hence the Q-factor should be within certain bandwidth-determined boundaries. Consequently, the required bandwidth shall pose a limit to how much noise improvement we can expect by using this method. The available $3 \mathrm{~dB}$ (peaking) bandwidth $\Delta \omega(10)[5]$ is found to be inversely proportional to the $\mathrm{Q}$-factor.

$$
\begin{gathered}
Q=\omega_{0} \frac{L_{\text {lead }}+L_{\text {explicit }}}{R_{T}}=\frac{\alpha}{R_{T}} \sqrt{\frac{L_{\text {lead }}+L_{\text {explicit }}}{C_{j}}} \\
\frac{\Delta \omega}{\omega_{0}} \approx \frac{1}{Q}
\end{gathered}
$$

\section{A PERFORMANCE COMPARISON BETWEEN RESONANT AND BROADBAND TIAS}

When designing a TIA for RoF links, dedicated resonant TIA designs can result in superior performances. There are three main reasons to opt for such an approach.

First of all, higher center frequencies can be targeted by a resonant TIA. This is possible because of the fact that one can resonate parasitics out at any given frequency as long as the required component values exist for the given frequency range. Additionally, the bandwidth for such a resonant tank should be sufficiently high to pass the signal band. Because of the fact that the components used in the design are assumed to be fast enough, this property is not covered in this article but alternative resonant receiver topologies can be devised to benefit from this property when needed.

Secondly, the SNR can be boosted significantly by adopting such a method. This increases the spectral efficiency that can be obtained because higher order modulation formats can be adopted for similar error rates. This is the main reason why we introduced a resonant design because it will result in high data rates even for a limited bandwidth. This SNR improvement is a nice side effect caused by the 
peaking of the input circuitry at the carrier frequency. The improvements will be limited by the maximum $Q$ value that can be obtained given a certain requirement on the signal bandwidth. However, the signal bandwidth for sub-6 GHz applications is typically limited since bandwidth is scarce and thus large sensitivity improvements can be expected by adopting series inductive peaking at the input.

A last advantage of this resonant technique is related to the limitation on the value that can be used for the feedback resistance (transimpedance limit, [6]) in broadband receivers. Consequently, there will be a limit to the output voltage. However, series inductive peaking at the input of the TIA boosts the transimpedance (11) and thus the output voltage. In equation (11), $Z_{T, 0}$ denotes the transimpedance at the resonance frequency (which is designed to coincide with the carrier frequency). The goal of the proposed resonant approach is to make sure that the SNR is significantly improved, resulting in $\left|Z_{T, 0}\right|>>$ $R_{F}$.

$$
Z_{T, 0}=-R_{F} \times \frac{I_{\text {in }}}{I_{P D}}\left(f_{0}\right)=-R_{F} \times Q \times \frac{1}{\alpha^{2}}
$$

\section{PROPOSED TIA DESIGN: A PROOF OF CONCEPT}

To illustrate the principle described in the previous section, a resonant TIA was designed with discrete components. For this proof-of-concept we targeted 150 MBaud communication of 64-QAM data modulated on a $4 \mathrm{GHz}$ carrier. The proposed TIA design that makes use of series inductive peaking is given in Fig. 4. This schematic includes the equivalent circuit of the PD (Fig. 1) and the amplifier with feedback resistor $R_{F}$.

An RF choke $L_{1}(8 \mathrm{nH})$ is added to bias the PD whereas $C_{1}(1 \mathrm{nF}), R_{\text {bias }}(51 \mathrm{k} \Omega)$ and $L_{2}(8 \mathrm{nH})$ are needed for obtaining the correct bias setting for the amplifier (where $V_{B B}$ provides $4 \mathrm{~V}$ ). The output part of the network consists of decouple capacitor $C_{2}(1 \mathrm{nF})$ and resistor $R_{\text {stab }}(100 \Omega)$. The latter is added to make the amplifier unconditionally stable [7]. Feedback resistance $R_{F}(330 \Omega)$ was chosen in function of the low-pass filtering introduced by the miller capacitance. Values for $C_{j}(445 \mathrm{fF}), R_{s}(7.09 \Omega)$ and $L_{\text {lead }}$ 
$(3.85 \mathrm{nH})$ resulted from S-parameter measurements of the $\mathrm{PD}$ with the required lead length. Equation

(8) was then used to calculate the desired value for decouple capacitor $C_{\text {block }}(1.5 \mathrm{pF})$.

\subsection{Transfer behavior}

A link consisting of a distributed feedback (DFB) laser and the proposed resonant PD/TIA combination was used to test the transfer behavior (Fig. 5). Frequency dependent effects of the laser were measured separately (with a high bandwidth photoreceiver) to compensate for the transmitter and make sure that the depicted transfer behavior only describes the PD/TIA combination rather than the whole link.

Simulations and measurements match very well for frequencies around the center frequency, over a range of nearly $2 \mathrm{GHz}$. However, an extra low frequency parasitic resonance is present that can be attributed to the fact that the RF choke $L_{1}$ (Fig. 4) has a finite value. In future designs, this peak can be damped by adding a resistor in series to inductor $L_{1}$. Discrepancies at higher frequencies can be attributed to the fact that the models for the devices used are no longer adequate at these higher frequencies. The peak around $6 \mathrm{GHz}$ is for example caused by a secondary resonance of the photodetector.

\subsection{Noise behavior}

Using the same link as in the transfer function measurements, a $4 \mathrm{GHz}$ sine wave is transmitted. The measured output spectrum shows not only the desired signal at the output but also a frequency dependent contribution of the noise (Fig. 6) where the frequency dependence resembles the transfer function (Fig. 5). It is important to remark that Fig. 6 was constructed under the assumption of a frequency flat Relative Intensity Noise (RIN) contribution. In practice, this is not the case and a maximum RIN contribution can be found close to the resonance frequency of the DFB laser $(6 \mathrm{GHz}$ for the given setup). This explains the discrepancy between the measured noise spectrum and the simulated noise spectrum. Noise shaping is clearly visible in the noise spectrum (Fig. 6) and this is the result from adopting a resonant approach. 
It can be seen that the RIN is the dominant noise effect for the given setup. Consequently, this link is not limited by the noise of the receiver. Nevertheless, the receiver will have a significant impact on how much the incoming noise deteriorates the signal. Using a resonant receiver will lower the noise contribution compared to a traditional broadband receiver, giving rise to an increase in SNR compared to a broadband TIA. This is caused by the fact that the RIN contribution experiences the previously mentioned noise shaping. The second most important noise contribution for the given setup (shot noise, introduced by the photodetector) experiences the same noise shaping.

\subsection{Constellation diagram}

Nearly the same link was used to do the communication tests. The only difference is that a $10 \mathrm{~dB}$ optical attenuator was added in between the DFB laser and the PD/TIA combination to lower the RIN and shot noise. The output of the resonant receiver was then sensed by a real-time scope (Fig. 7). It is shown in Fig. 7 that when a limited amount of equalization taps ( 3 taps) is added at the receiver, error-free (SNR $=35.8 \mathrm{~dB}$ ) 900 Mbps communication can easily be provided over an RoF link with a symbol rate of 150 MBaud.

\section{ESSENTIAL ROF SPECIFICATIONS: A CONCLUSION}

In the context of RoF links, there are several important specifications for the RAUs. Noise will be key in RoF to be able to transfer high order modulation formats. Traditional RAUs are, however, not yet optimized for the radio schemes used in RoF links and this article addresses this shortcoming by adopting a resonant design approach for the RAU receiver.

Furthermore, a massive amount of RAUs will be deployed in the case of 5G. Consequently, the cost, power consumption and occupied surface area should be small enough to make this technology scalable for 5G. The resonant TIA designed as a proof of concept has a footprint of $1.1 \mathrm{~cm} \mathrm{x} 1 \mathrm{~cm}$ and consumes $73.5 \mathrm{~mW}$ of power, which can be further optimized by integration on chip.

\section{ACKNOWLEDGMENT}


This work was partly supported by the European Commission in the framework of the 2020-ICT-2014-2

project Flex5Gware (Grant agreement no. 671563), by the UGent special research fund

(BOF14/GOA/034), by the Hercules project AUGE/13/01 and, by the iMinds loT research program. 


\section{FIGURES}

Fig. 1 Equivalent network of a PIN photodetector

Fig. 2 Broadband TIA: general structure

Fig. 3 Input circuitry TIA: Series inductive peaking

Fig. 4 TIA design using series inductive peaking

Fig. 5 Measured $\mathrm{S}_{21}$ compared to simulated $\mathrm{S}_{21}$

Fig. 6 Noise contributions (Resolution bandwidth $=10 \mathrm{kHz}$ )

Fig. 7 Constellation diagram: 64-QAM, 150 MBaud, $4 \mathrm{GHz}$ carrier, root raised cosine filter (roll-off =

$0.5)$ at receiver and transmitter (a): No equalization (b): 3 taps equalization 


\section{REFERENCES}

[1] V. A. Thomas, M. El-Hajjar and L. Hanzo, "Performance improvement and cost reduction techniques

for radio over fiber communications", IEEE Commun. Surveys Tuts., vol. 17, no. 2, pp. 627-670,

Second Quarter 2015

[2] V. J. Urick, K. J. Williams and J. D. McKinney, Fundamentals of Microwave Photonics, Wiley, 2015, pp. 25-26

[3] M. Neuhauser, H. M. Rein and H. Wernz, "Low-Noise, High-Gain Si-Bipolar Preamplifiers for $10 \mathrm{~Gb} / \mathrm{s}$ Optical-fiber links - Design and Realization", IEEE J. Solid-State Circuits, vol. 31, no. 1, pp. 24-29, Jan.1996

[4] E. Säckinger, Broadband Circuits for Optical Fiber Communication,Wiley, 2005, pp. 113-114

[5] F. Dunn, T. Rossing, W. M. Hartmann, D. M. Campbell and N. H. Fletcher, Springer Handbook of Acoustics, Springer, 2nd ed., 2015, pp. 532

[6] E. Säckinger, "The transimpedance limit", IEEE Trans. Circuits Syst. I, Reg. Papers, vol. 57, no. 8, pp. $1848-1856$, Aug. 2010

[7] D. M. Pozar, Microwave Engineering, Wiley, 2nd ed., 1997, pp. 612-622 


1
2
3
4
5
6
7
8
9
10
11
12
13
14
15
16
17
18
19
20
21
22
23
24
25
26
27
28
29
30
31
32
33
34
35
36
37
38
39
40
41
42
43
44
45
46
47
48
49
50
51
52
53
54
55
56
57
58
60

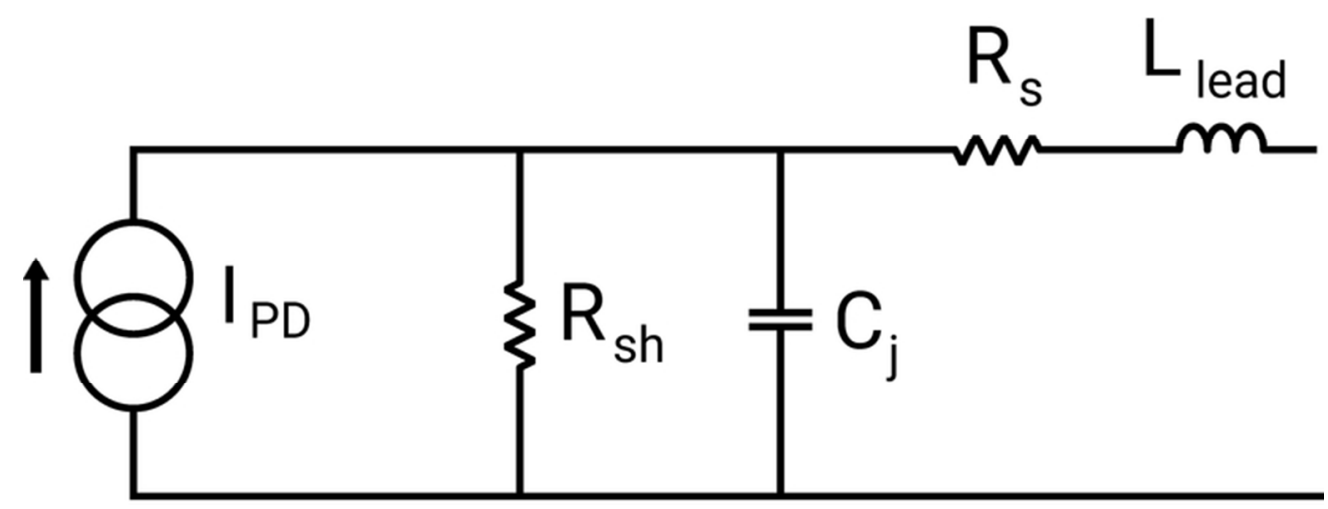

Fig. 1. Equivalent network of a PIN photodetector Fig. 1

$69 \times 26 \mathrm{~mm}(300 \times 300$ DPI $)$ 


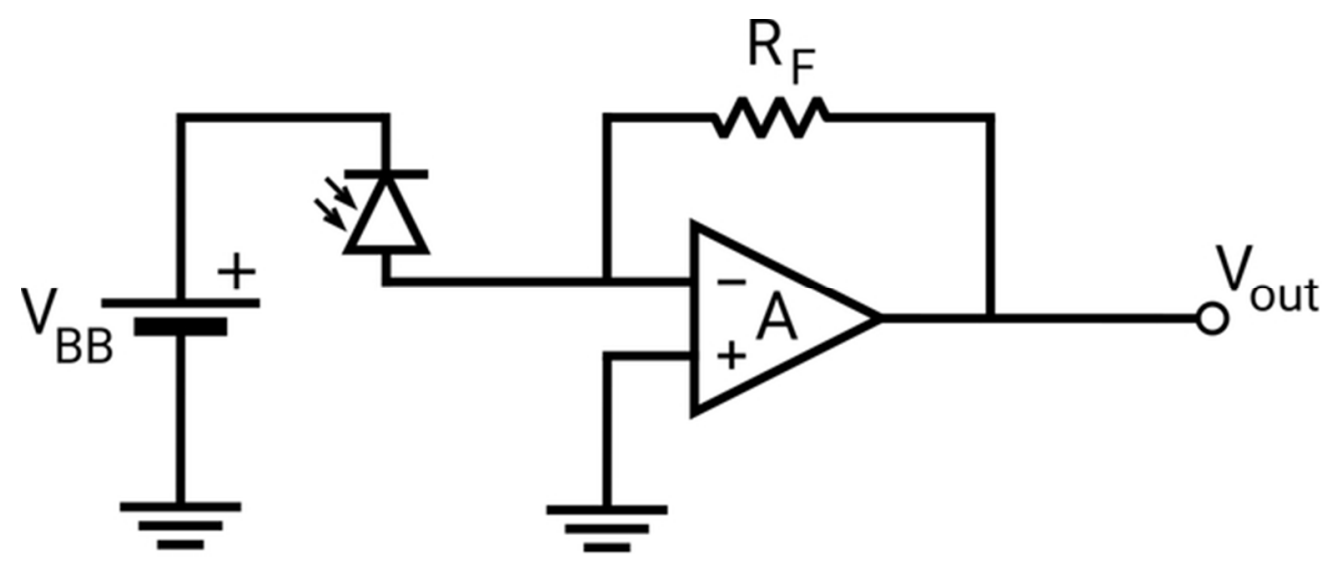

Fig. 2. Broadband TIA: general structure Fig. 2

$57 \times 23 \mathrm{~mm}(300 \times 300$ DPI $)$ 


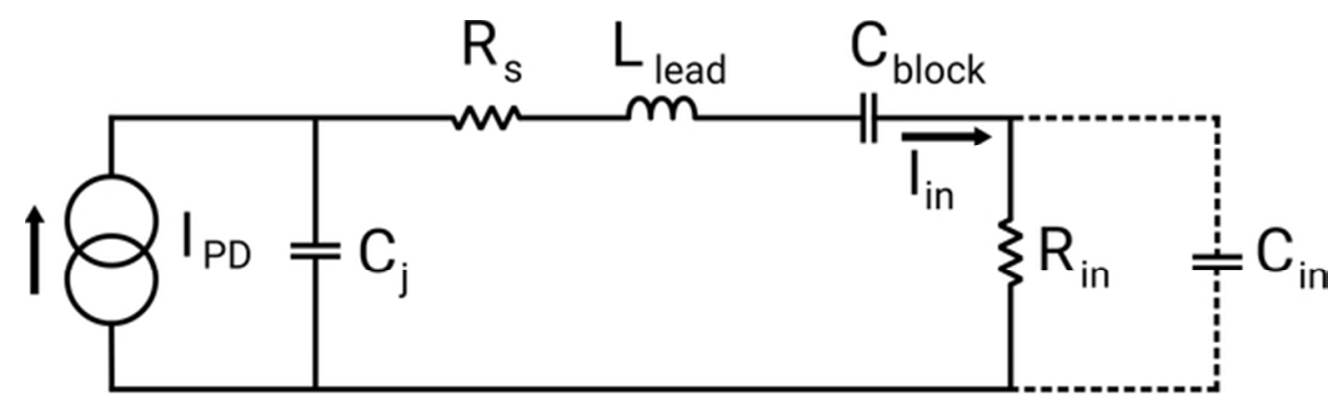

Fig. 3. Input circuitry TIA: Series inductive peaking Fig. 3

$55 \times 15 \mathrm{~mm}(300 \times 300$ DPI $)$ 


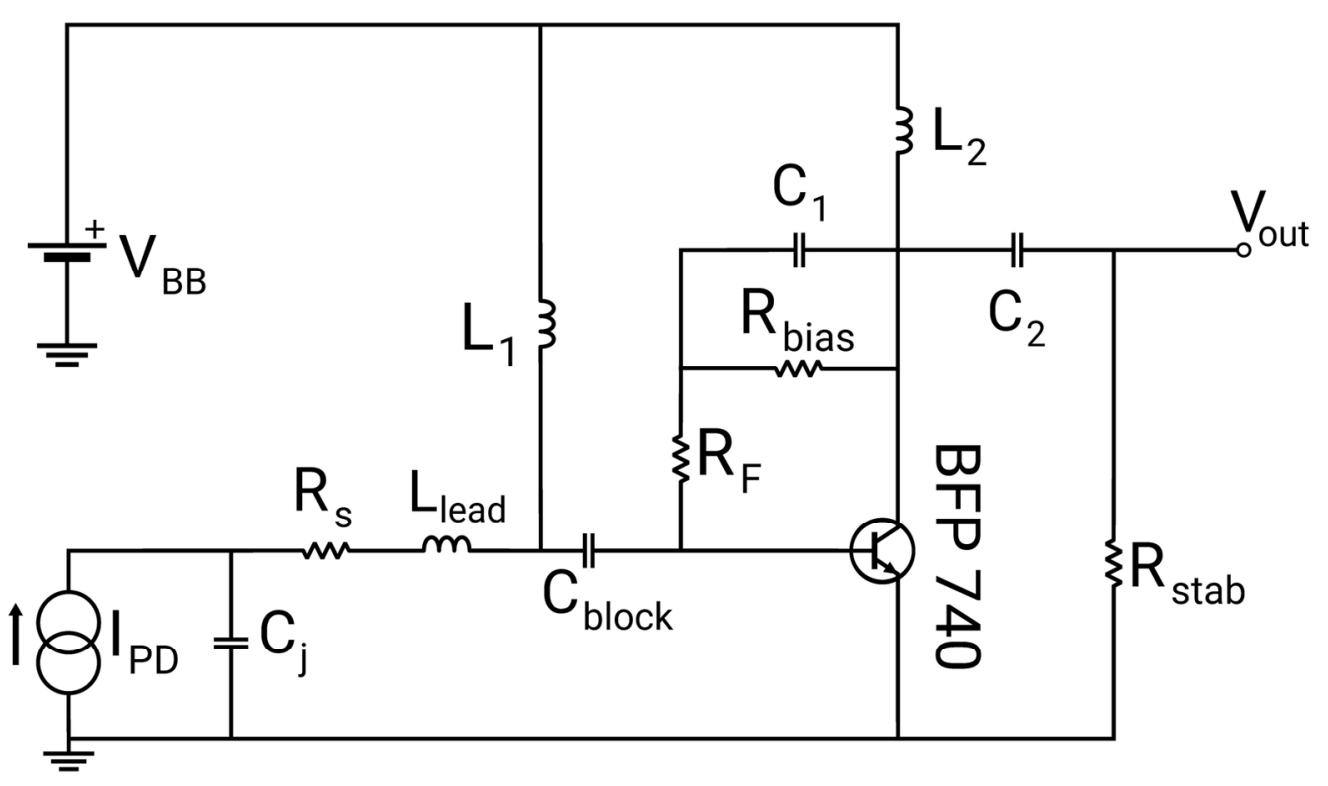

Fig. 4. TIA design using series inductive peaking Fig. 4

$152 \times 88 \mathrm{~mm}(300 \times 300$ DPI $)$ 


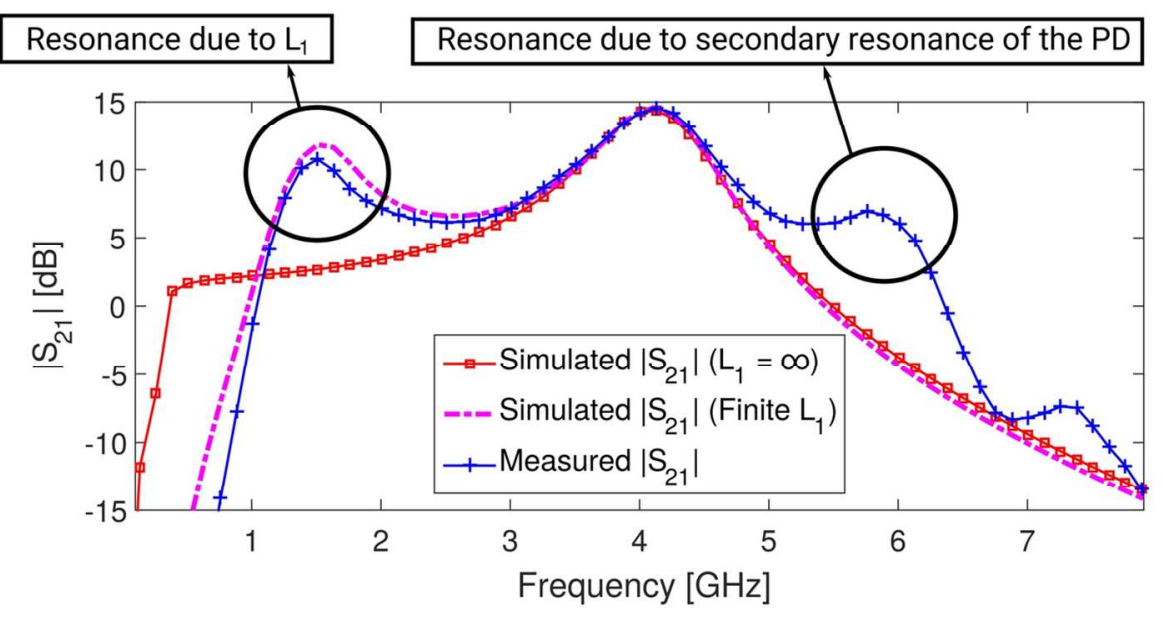

Fig. 5. Measured S21 compared to simulated S21

Fig. 5

$127 \times 58 \mathrm{~mm}(300 \times 300$ DPI $)$ 


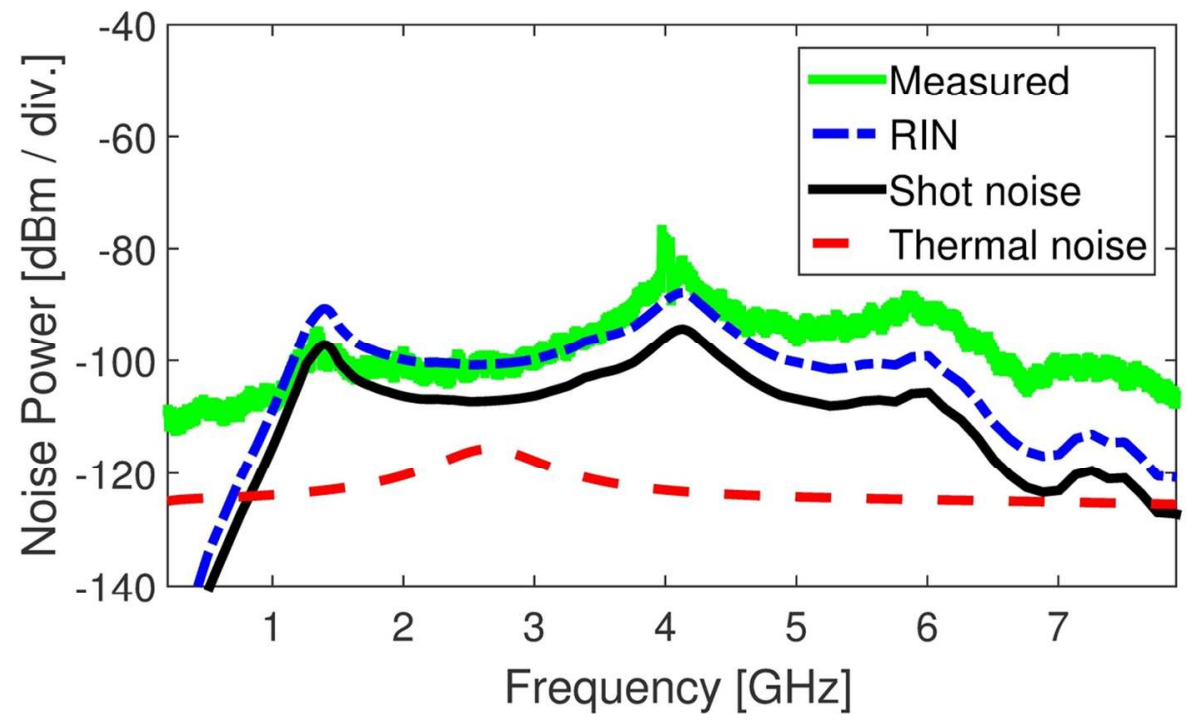

Fig. 6. Noise contributions (Resolution bandwidth $=10 \mathrm{kHz}$ )

Fig. 6

$114 \times 65 \mathrm{~mm}(300 \times 300$ DPI) 


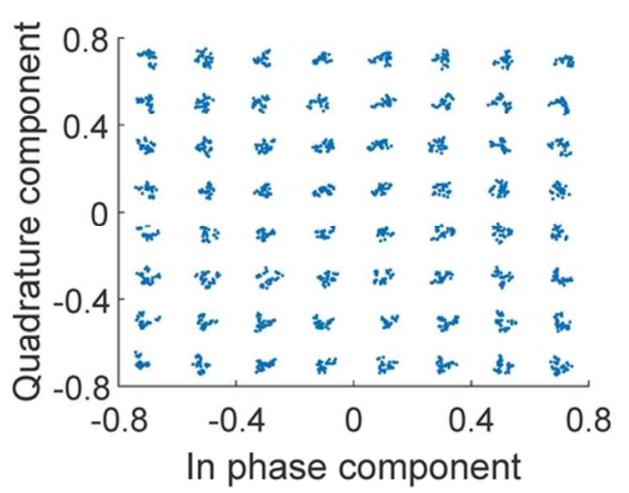

(a)

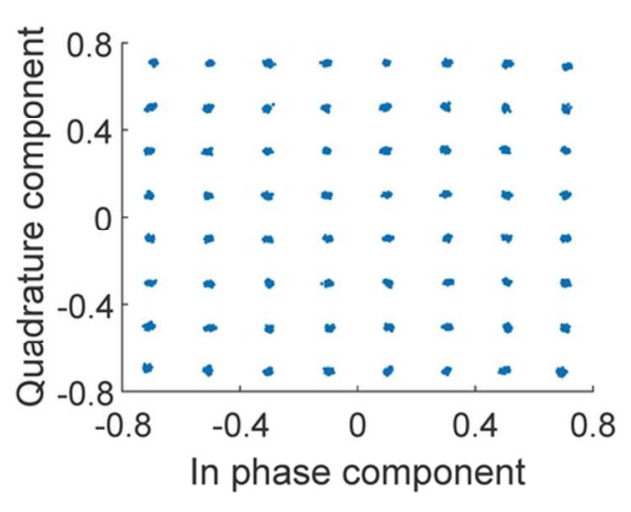

(b)

Fig. 7. Constellation diagram: 64-QAM, 150 MBaud, $4 \mathrm{GHz}$ carrier, root raised cosine filter (roll-off = 0.5) at receiver and transmitter (a): No equalization (b): 3 taps equalization

Fig. 7

$78 \times 34 \mathrm{~mm}(300 \times 300 \mathrm{DPI})$ 